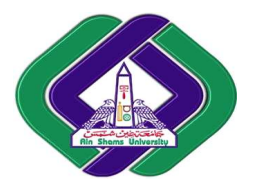

\author{
International Journal of Intelligent Computing and \\ Information Science
}

\title{
MRI BRAIN IMAGE SEGMENTATION BASED ON CASCADED FRACTIONAL-ORDER DARWINIAN PARTICLE SWARM OPTIMIZATION AND MEAN SHIFT
}

\begin{abstract}
H. Ali
M. Elmogy

E. El-Daydamony

A. Atwan

Information TechnologyDepartment,Faculty of Computers and Information, Mansoura University - Egypt.

Hala_ahmed703@yahoo.com,_melmogy@mans.edu.egrEman.8.2000@gmail.com_Atwan_2@yahoo.com

Abstract:Image segmentation is an initiative with massive interest in many imaging applications, such as medical images and computer vision. It is considered as a challenging problem, so we need to develop an efficient, fast technique for medical image segmentation. In this paper, the proposed framework is based on two segmentation methods: Fractional-order Darwinian Particle Swarm Optimization (FODPSO) and Mean Shift segmentation (MS). FODPSO is a favorable method for specifying a predefined number of clusters and it can find the optimal set of thresholds with a higher between-class variance in less computational time. In the pre-processing phase,the MRI image is filtered and the skull is removed. In the segmentation phase, the result of FODPSO is used as the input to MS. Finally, we make a validation to thesegmented image. We compared our proposed system with some state of the art segmentation techniques using brain benchmark data set. The experimental results show that the proposed system enhances the accuracy of the MRI brain image segmentation.
\end{abstract}

Keywords:Medical Image Segmentation, MRI Brain Images, Multi-levelSegmentation,Fractional-order Darwinian Particle Swarm Optimization (FODPSO), Mean Shift (MS).

\section{Introduction}

Segmentation can be defined as the operation of dividing images into constituent sub-regions. The group of segments or sub-regions is the result of image segmentation that collectively covers the whole image or a set of contours derived from the image. Color, intensity, or textures are some considerations or computed properties for classifying the pixels in some regions. Adjacent regions are significantly different with respect to the same characteristic(s) [1]. The manual segmentation is possible, but it takes much time. In addition, there is a significant difficulty in reproducing a manual segmentation result due to the low level of confidence ascribed that we suffer accordingly. Therefore, automatic segmentation methods are afavorite and an active research area. The complexity of the segmentation arises from the various characteristics of the images. Therefore, medical image segmentation is considered as a challenging task [2].Image segmentation divides digital images into non-overlapping regions. It extracts significant and meaningful information from the processedimages.In addition, thenumerousanalysis can be performed to extract critical areas from the images[3]. Magnetic Resonance Imaging (MRI) is the most commonly used technique for evaluating the anatomical of human brain structures. MRI provides a comprehensive vision of what happen in patient's brain. It consists of the typicalstructures of brains, 
such as gray matter (GM), white matter (WM), cerebrospinal fluid (CSF), and damageregions. They are presentedin single common structures or overlapped areas [4].WM, GM, and CSF need theaccurate measurement for the quantitative pathological analyzes. Segmentation of the MRI brain image data is a goal that is requiredto process these regions[5].

There are several image segmentation techniques proposed and available for medical application. These methods are chosen with respect to the specific applications and different imaging modalities. The performance of segmentation algorithms is still challenging because there are several imaging problems, such as noise, partial volume effects, and motion. Some of these methods, such as thresholding methods, region-growing methods, and clustering methods, were studied by many researchers [6, 7]. Thresholdingis considered as one of the main techniques of the medical image segmentation. It depends on separating pixels into different classes according to their gray levels. Partitioning the scalar image intensities to a binary is made by using thresholding approaches. The operation of segmentation is then performed by groping all pixels with the intensity value that have greater value than the threshold into one class, and all other pixels into another class. Multi-thresholdingcan be determined by processing the threshold with many values instead of only one value. The main restriction of the multi-thresholdingis that it deals only with two classes in its simplest form, and it cannot be applied to multi-channel images $[6,7]$. In addition, the spatial characteristics of an image in the thresholding method do not typically be taken into consideration. Therefore, it is susceptible to noise and intensity inhomogeneities, which can occur in MRI images.

Clustering algorithms are the most common used techniques of image segmentation. We can define clustering as an unsupervised learning technique, which needs the user to determine the number of clusters in advance to classify pixels [8]. As a result, the cluster is a collection of both similar pixels and dissimilar to the pixels belonging to other clusters [9]. There are two ways can be used in clustering algorithms: partitioning and grouping pixels [10]. In partitioning type, the whole image can be divided into smaller clusters in a successive way by the clustering algorithm. Whereas in the grouping type, the algorithm starts with each element as a separate cluster and gather them in successively larger clusters. The pixels are grouped together based on some assumptions that decide how to group them preferably. The most commonly used methods also for the image segmentation is the thresholding that segment images into two or more clusters. Thresholding techniques have two divisions: optimal thresholding methods and property-based thresholding methods. Algorithms in the former group make the threshold classes on the histogram achieved desired characteristics for obtaining the optimal thresholds. The thresholds selection can be got by optimizing an objective function. Some selected property of the histogram is measured by the latter group algorithms for detecting the thresholds. The multilevel thresholding needs fast methods that make the property-based thresholding methods more suitable for it. However, the number of thresholds for these approaches is hard to determine and needs to be specified in advance [11].

In this paper, we concentrate on both clustering and multilevel thresholding methods for medical brain MRI image segmentation. We conducted our experiments by using the most used multilevel thresholding and clustering techniques: POS, DPSO, FODPSO+MS, FCM and MS. This paper is divided into five sections as follows. Section 2 introduces the basic concepts of some different medical image segmentation systems. Section 3 presents the current related work of some different medical image segmentation systems. In Section 4, the proposed medical image segmentation system based on our proposed Cascaded Fractional-order Darwinian Particle Swarm Optimization and Mean Shift. The experimental results and discussion of these results on two different benchmark data sets are discussedin Section 5. Finally, we present the conclusion and the future work in Section 6. 


\section{Basic Concepts}

deal with agray image because color image takes more computation time. For each image, there are $\mathrm{L}$ intensity levels, which are in the range of $\{0,1,2, \ldots, L-1\}$. Then,we can define the probability distribution An efficient way to perform image analysis is to use multi-level segmentation techniques. However, the selection of a robust optimum n-level threshold is required to be automatic. In the following discussion, a more accurate formulation of the problem is introduced.

\subsection{Image Thresholding}

Image analysis can be performed in an efficient way by using multi-level thresholding segmentation techniques. The essential challenge in the image segmentation is the selection of the optimum n-level threshold. However, the selection of the optimum n-level threshold is required to be automated. This section presents a more precise formulation of the problem, introducing some basic notation.

In our proposed system, we as [12]:

$$
\mathrm{p}_{\mathrm{i}}=\frac{\mathrm{h}_{\mathrm{i}}}{\mathrm{N}}, \Sigma_{\mathrm{i}=1}^{\mathrm{N}} \mathrm{p}_{\mathrm{i}}=1
$$

wherei represents a particular intensity level, i.e., $1 \leq i \leq L-1$. The total number of the pixels in the image is N.The number of pixels can be represented by $h_{i}$ for the corresponding intensity level $i$. In other words, image histogram is represented by $h_{i}$, which can be normalized and considered as the probability distribution $p_{i}$ for component of the image.The total mean (i.e., combined mean) can be simply computed as:

$\mu_{T}=i p_{i}$

The generic n-level thresholding can be derived from the 2-level thresholding in which n-1 threshold levels $t_{\mathrm{j}}, \mathrm{j}-1, \ldots, \mathbf{n}-\mathbf{1}$, are necessary and where the operation is performed as expressed below in Eq.

$$
F(x, y)=\left\{\begin{array}{cc}
0 & f(x, y) \leq t_{1} \\
\frac{1}{2}\left(t_{1}+t_{2}\right), & t_{1}<f(x, y) \leq t_{2} \\
\vdots & \\
\frac{1}{2}\left(t_{n-2}+t_{n-1}\right), & t_{n-2}<f(x, y) \leq t_{n-1} \\
L & f(x, y)>t_{n-1}
\end{array}\right.
$$

The image isrepresentedby $\mathrm{x}$, which is the width $(\mathrm{W})$ of the image, and $\mathrm{y}$, which is the height $(\mathrm{H})$ of the image. Then, the size can be represented by $\mathrm{H} \times \mathrm{W}$ denoted by $\mathrm{f}(\mathrm{x}, \mathrm{y})$ with $\mathrm{L}$ intensity gray levels. In this situation, the pixels of a given image will be divided into $n$ classes $\left(D_{1}, \ldots, D_{n}\right)$. It may represent

multiple objects or even specific features on such objects (e.g., topological features).

The method that maximizes the between-class variance is used for obtaining the optimal threshold.It is the most efficient computational method that can be generally defined by: 


$$
\sigma_{\mathrm{B}}^{\mathrm{a}}=\sum_{\mathrm{j}=1}^{\mathrm{n}} \mathrm{W}_{\mathrm{J}}\left(\mu_{\mathrm{j}}-\mu_{\mathrm{T}}\right)^{2},
$$

where $\mathrm{j}$ represents a particular class in such a way that $W_{I}$ and $\mu_{j}$ are the probability of occurrence and the mean of the class $j$, respectively. The probabilities of occurrence $W_{J}$ of classes $D_{1}, \ldots, D_{n}$ are given by:

$$
\mathrm{W}_{\mathrm{J}}=\left\{\begin{array}{c}
\sum_{i=1}^{t_{j}} p_{i, j}=1 \\
\sum_{i=t_{j-1}+1}^{t_{j}} p_{i, 1} 1<j<n_{y} \\
\sum_{i=t_{j-1}+1}^{L} p_{i j} j=n_{y}
\end{array}\right.
$$

The mean of each class $w_{\mathrm{J}}$ can then be calculated as:

$$
\mu_{j}=\left\{\begin{array}{c}
\sum_{i=1}^{t_{j}} \frac{i p_{i}}{W_{j}} j=1 \\
\sum_{i=t_{j-1}+1}^{t_{j}} \frac{i p_{i}}{W_{j}}, 1<j<n, \\
\sum_{i=i_{j-1}+1}^{L} \frac{i p_{i}}{W_{j}}, j=n,
\end{array}\right.
$$

In other words, the n-level thresholding problem is limited to an optimization problem. It searches for the thresholds $t_{\mathrm{j}}$ that make maximization for the objective function (i.e., a fitness function) defined as:

$$
\varphi=\max _{1<t_{1}<\ldots<t_{n-1}<L} \sigma_{E}^{2}\left(t_{j}\right)
$$

As the number of threshold levels increases, this optimization problem involves a much larger computational effort. It makes us think of the question: which type of methods that the researchercan use for solving this optimization problem for real-time applications? [12]. FODPSO is an example of such methods that recently presented. FODPSO is a new version that derived from the DPSO. To control the convergence rate of FODPSO, the fractional calculusis used to solve this kind of problems [13].

\subsection{The Mean Shift (MS) Algorithm}

Mean Shift Clustering is an example of an unsupervised clustering techniquethat does not require prior knowledge, such as the number of the data cluster. It is an iterative method that starts with an initial estimated [14]. MS segmentation is used for making concatenation for both the spatial and range domains of an image. In addition, it is used for identifying modes in this multidimensional joint spatial- 
range feature space. The bandwidth parameter (the value of kernel size) is free and is not restricted to a constant value. Several methods are used for estimating a single fixed bandwidth. Over-clustering and under-clustering arise from the chosen value of the bandwidth. The too small value of the bandwidth produces over clustering and also the too large value of bandwidth provide critical modes can be merged under-clustering. When the feature space has significantly different local characteristics across the space, the use of a single fixed bandwidth has the drawback that it can produce under- or overclustering [15].

\section{Related Work}

Image segmentation plays a significant role in the field of medical image analysis. Thresholdingis considered as one of the main techniques of the medical image segmentation. Therefore, many researchers have proposed many segmentation techniques for obtaining optimal threshold values based on multi-thresholding method for image segmentation. For example, Ghamisi et al. [12] presented two novel methods for images segmentation to identifying the n-1 optimal forthe n-level threshold. The FODPSO and Darwinian Particle Swarm Optimization (DPSO) are proposed for image segmentation. Delineating multilevel threshold and the disadvantages of preceding methods in terms of limitation of the local optimum and high CPU process time is solved by using these two methods [12]. The efficiency of other well-known thresholding segmentation methods is compared with their proposed methods. When taking into consideration a number of different measures, such as the fitness value, STD, and CPU,their experimental results showed that their proposed methods superior to other methods. On the other hand, they did not handle real-time image segmentation.

Ghamisi et al. [13] introduced two main segmentation approaches for classification of hyperspectral images. They used FODPSO and MS segmentation techniques. The support vector machine (SVM) is used for classifying the output of these two methods. In their proposed system, in the beginning, the input image with (B bands) entersto the FODPSO to perform segmentation. Second, the output of FODPSO is supplied to MS as input to make segmentation to the (B bands) image. Finally, the classification process of ( $\mathrm{B}$ bands) to produce $(1$ band) image is doing by using SVM.The main disadvantage of MS is the tuning size of the kernel, and the obtained result may considerably be affected by the kernel size.Hamdoui et al. [16] proposed an approach that known as Multithresholding based on Modified Particle Swarm Optimization (MMPSO). Theyimplemented their proposed method for segmenting images based on Particle Swarm Optimization (PSO)to identify a multilevel threshold. They mentioned that their proposed method is suitable for complex gray-level images. Their results indicated that the MMPSO is more efficient than PSO and GA. They did not classify MRI medical images because it is a low computational complexity and leave it as a future work.Jin et al. [5] proposed a multispectral MRI brain image segmentation algorithm. This algorithm based on kernel clustering analysis. The algorithm is called as multi-spectral kernel based fuzzy c-means clustering (MS-KFCM). In their proposed system, MRI T1-weighted and T2-weighted brain image are filtered and then make a selection to the features as the input data. The separation improvement of the input data is doing by mapping the input data to a high dimensional feature space. The output of fuzzy c-means (FCM) clustering is used as the initial clustering center of MS-KFCM. The performance of MS-KFCM is better than FCM and KFCM, but FCM and KFCM are similar in the performance. The advantage of using the multi-spectral image segmentation is to achieve higher accuracy than to use single-channel image segmentation.

Mangala and Suma [17] presented brain MR image segmentation algorithm that is called Fuzzy Local Gaussian Mixture Model (FLGMM). They removed noise by applying Gaussian filter.They handled the 
bias field estimation by using BCFCM. Second, all techniques initialized by using K-Means. Then, they used FLGMM to make segmentation to the processed image. The segmentation accuracy was measured by the Jaccard similarity (JS). The value of JS ranges from 0 to 1 , and a higher JS represents segmentation that is more accurate. They did not deal with reducing the computational complexity and improving the robustness.

Bandhyopadhyay and Paul [18] proposed a system for diagnosing the brain tumor. The first stage of their proposed system is the registration processthat is appliedto the adjacent layers of the brain. In the second stage, they made fusion between registered images to obtain ahigh-quality image for the segmentation. Finally, they used improved K-means algorithm with adual localization methodology toperform the segmentation process. They mentioned that they wouldconcentrate on $3 \mathrm{D}$ modeling for segmentation of the image and detection of the tumors.

AbdelMaksoud et al. $[19,20]$ proposed an accurate system for detecting the brain tumor with minimal execution time. Their system is based on hybrid clustering techniques. Two hybrid techniques have been introduced in their proposed medical image segmentation system. The first one is based on k-means and fuzzy c-means (KFCM). The second is based on k-means and particle swarm optimization (KPSO). Theirsystem consists of four phase. In clustering stage, they were used KFCM or KPSO to detect atumor in the image. Finally, thresholding and active contour level set are used for segmentation. In future work, they seek for detecting the brain tumor by using 3D Slicer.In this paper, we propose a medical image segmentation system based on the FODPSO and MS techniques. The principal objective is to accurately detect the brain structure (WM, GM, and CSF) in minimal execution time. We consider both the accuracy and the minimum execution time in our proposed system. In the preprocessing stage, we applied the median filter to enhance the entire image quality and removed the skull from the processed image. This stage reduces both the processing time and the used amount of memory. In segmentation stage, we use FODPSO as a pre-pressing step to MS to solve the drawbacks of MS. Finally, we make a validation to segmented image with the ground truth as illustrated in experimental results.

\section{The Proposed MRI Image Segmentation System}

There are many medical image segmentation systems that are used for detecting brain structure and tumor. All of these systems are not equal in accuracy and in execution time. Therefore, our goal is to build a robust segmentation system to deal with the brain images.As all thresholding-based methods, FODPSO segmentation suffers from two main disadvantages. First, it cannot handle inhomogeneity. Second, it fails when the intensity of the object of interest does not appear as a peak in the histogram. In the MS method, the size of the kernelneeds to be tuned by the user [13]. The tuning may be a difficult task, and the final results may be dramatically affected.The proposed medical image segmentation system consists of three main phases: pre-processing, segmentation, and validation, as shown in Figure 1. We take into account the accuracy and the time. In the preprocessing stage, we used the median filter and brain extractor tool for skull stripping from the processed image. In the segmentation phase, we make integration between MS and FODPSO that takes all advantages of them. Finally, validation is performed on the proposed system and the ground truth. 


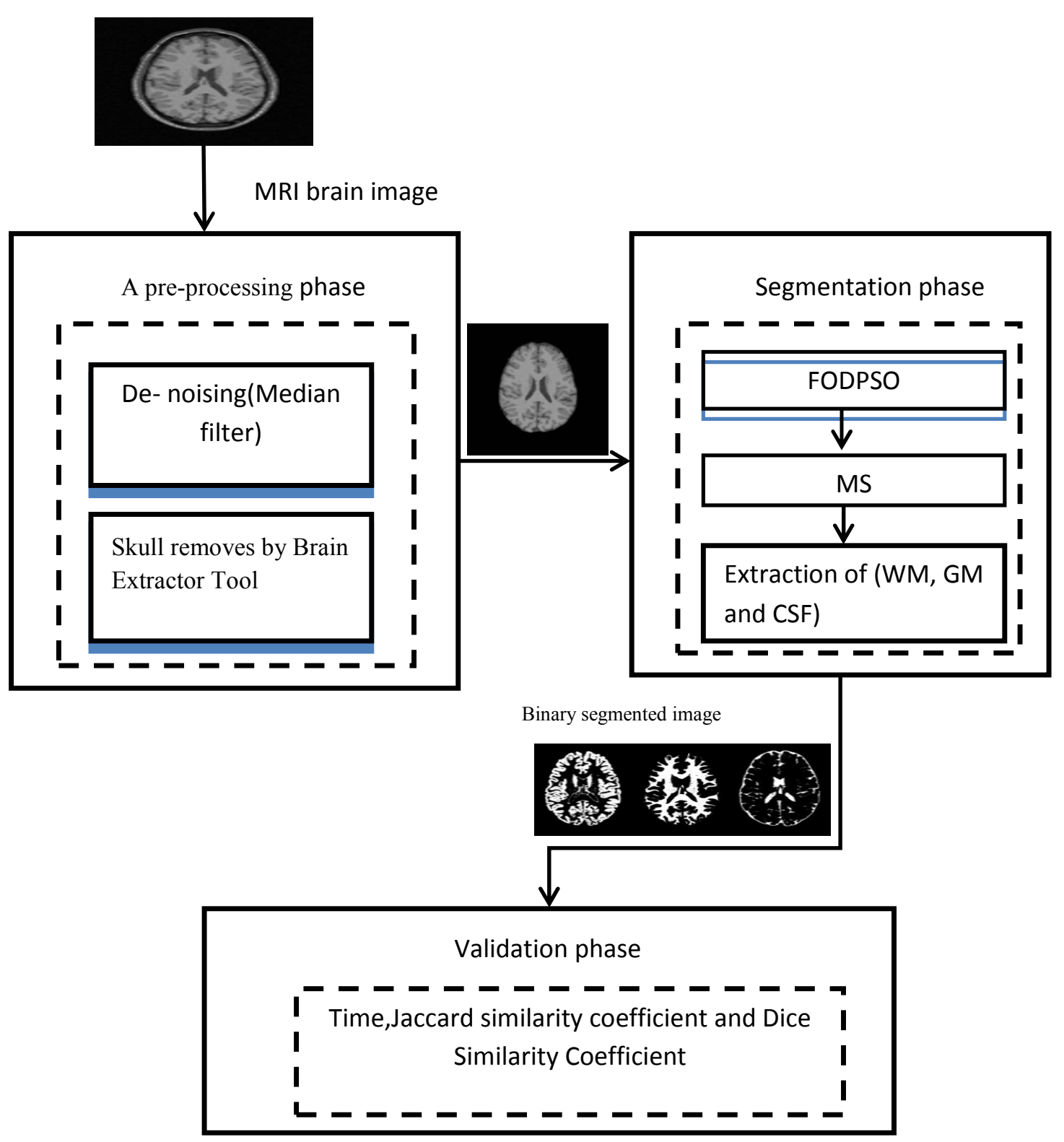

Figure 1: The block diagram of the proposed framework.

\subsection{The PreprocessingPhase}

The main purpose of this stage is to improve the image quality and remove the noise.The pre-processing stage consists of the de-noising and skull stripping sub-stages. The de-noising is necessary for medical images to be sharp, clear, and eliminatingnoise and artifacts. MRI images are usually affected by Gaussian and Poisson noise [21]. By using a median filter, all the pixel values are sorted from the window into numerical order, then the processed pixel is replaced by the median of the pixel values. Linear filtering is not better as median filtering for removing noise in the existence of edges [22]. On the other hand, the skull and the background of the image are removed while they do not contain any useful information. Decreasing the amount of the memory usage and increase the processing speed are 
done by removing unhelpful information, such as background, skull, scalp, eyes, and all other structures. Skull removal is done by using BET(Brain Extractor Tool) algorithm [23].

\subsection{The Segmentation Phase}

In this stage, we make integration between MS and FODPSO to take the advantages of these segmentation techniques. First, an input MRIbrain image will be segmented by FODPSO. Then, the output of this step will be segmented again by MS. In other words, The result of FODPSO is used as input to MS. The FODPSO is a favorable method for specifying a predefined number of clusters. It can find the optimal set of thresholds with a higher between-class variance in less computational time. Therefore, we extract brain structure (WM, GM, and CSF) from segmented image to binary image then the proposed system is validated in the next phase.

\subsection{The ValidationPhase}

In this stage, the result of the image segmentation with the proposed clustering techniques was compared to the ground truth as illustrated in the experimental results. The calculated measures are time, Jaccard similaritycoefficient, and Dice similarity coefficient. The performance of the segmented images is illustrated in the experimental results in details and how to compute each of the performance measures.

\section{The Experimental Results and Discussion}

In order to measure the performance of our proposed image segmentation approach, the proposed algorithm is implemented in MATLAB R2011a on a Core(TM) 2 Due, $2 \mathrm{GHz}$ processor, and 4GB RAM.We used two standard datasets. The first data set is BRATS [24] database from Multimodal Brain Tumor Segmentation. It consists of 30 glioma patients with multi-contrast MRI scans (both low-grade and high-grade, and both with and without resection) along with expert observation for "active tumor" and "edema". For each patient, there are many available types of images, such as T1, T2, FLAIR, and post-Gadolinium T1 MRI images.This database contains 81 images and has ground truth images to compare the results of our method with them. These images are got from Brain Web Database at the McConnell Brain Imaging Centre of the Montreal Neurological Institute, McGill University. The second is the Brain Web [25] database. It contains phantom and simulated brain MRI data based on two anatomical models:normal and multiple sclerosis. For both of these models, the data volumes of the full 3-dimensional data are emulating by using the three sequences (T1-, T2-, and protondensity- (PD-) weighted). On the other hand, there is a variety of slice thicknesses, noise levels, and non-uniformity levels of intensity. It is a T1 modality, $1 \mathrm{~mm}$ slice thickness. This dataset consists of 152 images.

\subsection{Measuring the Segmentation Performance}

To provide apropercomparison between the tested methods, we use different performance measures, such as:

1. Jaccard similarity coefficient $[\mathbf{2 6}, \mathbf{2 7}]$ : It is a widely used overlap measure, which is public and used usually as similarity indices for binary data. The area of overlap JSC is computed between the segmented image $S_{1}$ and the gold standard image $S_{2}$ as shown in equation. 


$$
I S C=\operatorname{vol}\left(S_{1} \cap S_{2}\right) / \operatorname{vol}\left(S_{1} \cup S_{2}\right)
$$

2. Dice similarity coefficient [26, 27]: It measures the number of the extent of spatialoverlap between two binary images. It is the most widely used for measuring the performance of segmentation. Its values range between 0 and 1 if the value is zero there is no overlap. If the value is one, this means a good agreement.The Dice coefficient is defined as:

$$
D=2 \operatorname{vol}\left(S_{1} \cap S_{2}\right) / \operatorname{vol}\left(S_{1}+S_{2}\right)=2 J S C /(1+J S C)(9)
$$

Table 1: The parameters of FODPSO

\begin{tabular}{|l|l|l|l|l|l|l|l|l|l|l|l|}
\hline $\begin{array}{l}\text { Parameters } \\
\text { method }\end{array}$ & $\mathbf{I}_{\mathrm{T}}$ & $\mathbf{N}$ & $\rho_{1} \rho_{2}$ & $\Delta v$ & $N_{\max }$ & $N_{\min }$ & $N^{S}$ & $N_{\max }^{S}$ & $N_{\min }^{S}$ & $N_{\text {Ril }}$ & $\propto$ \\
\hline FODPSO & $\mathbf{1 5 0}$ & $\mathbf{3 0}$ & $\mathbf{0 . 8}$ & $\mathbf{3}$ & $\mathbf{5 0}$ & $\mathbf{1 0}$ & $\mathbf{4}$ & $\mathbf{6}$ & $\mathbf{2}$ & $\mathbf{1 0}$ & $\mathbf{0 . 6}$ \\
\hline
\end{tabular}

In Table 1, we listed the main parameter of FODPSO. The number of iterations is IT; $\mathrm{N}$ is the population,the coefficients $\rho_{1 \text { and }} \rho_{2}$ are weights, which control the global and individual performance. The parameter $\propto$, commonly known as the fractional coefficient, will weigh the influence of past events in determining a new velocity, $0<0<1$. The number of swarms is $\mathrm{N}^{z}$ where $\mathrm{N}_{\max }^{z}$ represents the maximum number of allowed swarms. $\mathbf{N}_{\min }^{\mathrm{E}}$ represents the minimum number of allowed swarms. The number of particles is described by $N_{k i l l}$, no enhancement in fitness means that the number of particles was deleted by the swarm over a period.

\begin{tabular}{|c|c|c|c|c|}
\hline $\begin{array}{l}\text { Data } \\
\text { Set }\end{array}$ & Original & BET & FODPSO+M & Truth/normal \\
\hline $\bar{n}$ & & $\begin{array}{l}\text { The skull is } \\
\text { already } \\
\text { removed }\end{array}$ & & \\
\hline ֶิ & & & & $S_{5}$ \\
\hline
\end{tabular}

Table 2: The main steps of the proposed framework.

Table 2 shows the main stages of the proposed method. The first stage is the skull removal that performed by using BET algorithm [23]. The second stage usesthe FODPSO algorithm combined withthe MS algorithm. The output of FODPSO is supplied as an input to MS. MS needs to enter the parameters of the bandwidth, the threshold, and the number of clusters K. By doing the experiments on all images of the two data sets using the MS, we found that the best results in image clusters can be got 
if bandwidth $=0.2$. By decreasing the bandwidth for the same threshold, it processes the images in less time.

Table 3: The comparison between five different segmentation techniques on the two tested datasets.

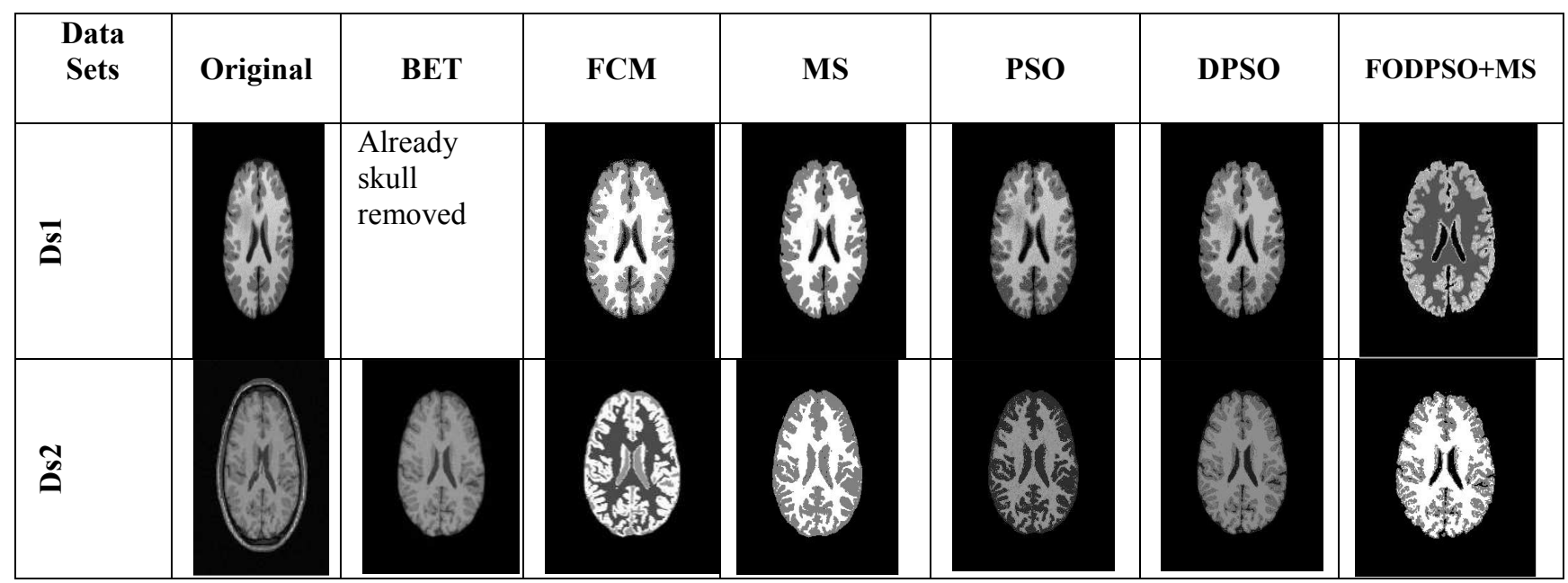

Table 4: The mean errors for the Jaccardand the Dice similarity coefficientsfor DS1.

\begin{tabular}{|l|l|l|l|l|l|}
\hline \multicolumn{7}{|c|}{ Segmentation techniques for DS1 } \\
\hline \multicolumn{7}{|c|}{ FCMMS PSO DPSOFODPSO+MS } \\
\hline JSC & 0.9136 & 0.9178 & 0.9312 & 0.9433 & 0.9821 \\
\hline Dice & 0.9548 & 0.9571 & 0.9644 & 0.9708 & 0.9910 \\
\hline time & $11.47670 \mathrm{Sec}$ & $0.785911 \mathrm{sec}$ & $31.3395 \mathrm{Sec}$ & $30.9704 \mathrm{Sec}$ & $12.8960 \mathrm{sec}$ \\
\hline
\end{tabular}

Table 5: The mean errors for the Jaccardand the Dice similarity coefficientsfor DS2.

\begin{tabular}{|l|l|l|l|l|l|}
\hline \multicolumn{5}{|c|}{ Segmentation techniques for Ds2 } \\
\hline \multicolumn{2}{|c|}{ FCM } & \multicolumn{1}{c|}{ MS } & PSO & \multicolumn{1}{c|}{ DPSO } & \multicolumn{1}{c|}{ FODPSO+MS } \\
\hline JSC & 0.9223 & 0.9223 & 0.9389 & 0.9478 & 0.9825 \\
\hline Dice & 0.9596 & 0.9596 & 0.9685 & 0.9732 & 0.9912 \\
\hline time & $10.228735 \mathrm{sec}$ & $0.654596 \mathrm{sec}$ & $28.1894 \mathrm{sec}$ & $24.8010 \mathrm{sec}$ & $12.2559 \mathrm{sec}$ \\
\hline
\end{tabular}

In Tables 4 and 5, we measure the mean errors in the two tested data sets by using the JSC and Dice. We can observe that our proposed technique (FODPSO+MS) gives the best result than any other tested techniques. 


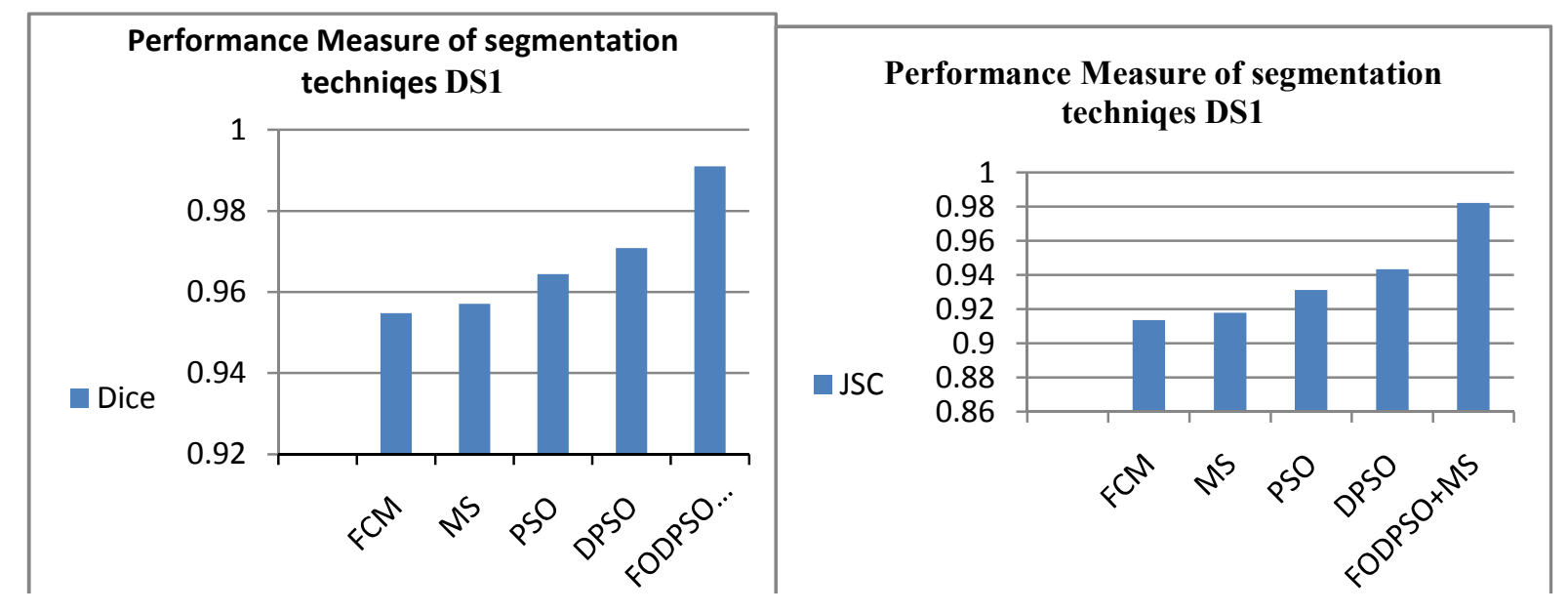

Figure 2: The performance measure of the segmentation techniques in seconds for DS1

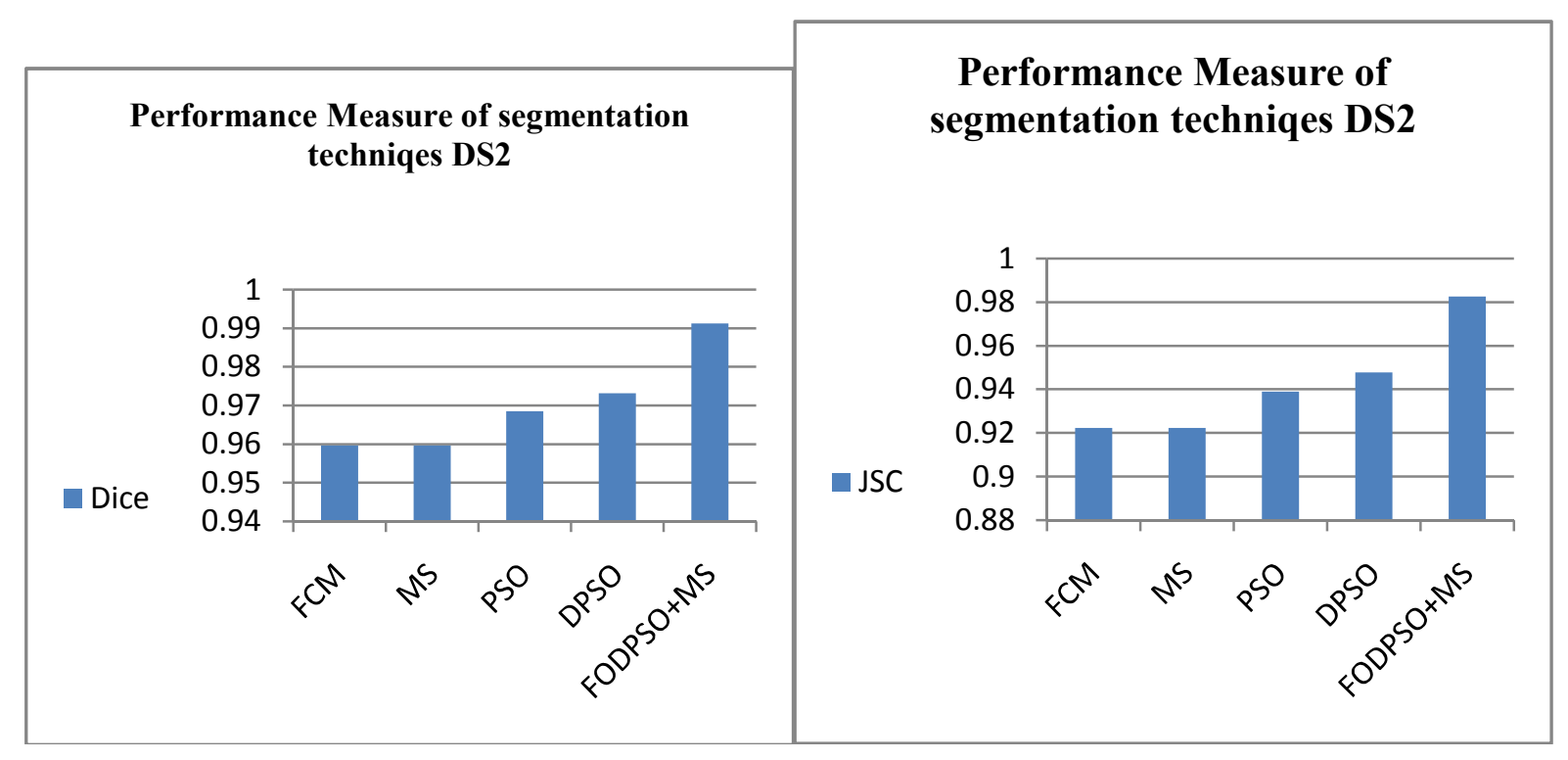

Figure 3: The performance measure of the segmentation techniques in seconds for DS2

\section{Conclusion}

The medical images contain unknown noise, and therefore it is hard to achieve acceptable performance for the segmentation process. The proposed approach is based on the combination of FODPSO and MS techniques. FODPSO is a very robust approach to finding the predefined number of clusters. It is able to find the optimal set of thresholds with a higher between-class variance in less computational time. In the proposed approach, the result of FODPSO is used as the input to MS to develop a pre-processing method for the classification. The main difficulty of MS is tuning the size of the kernel, and the obtained result may be affected by the kernel size. Results indicate that the use of both segmentation methods can overcome the shortcomings of each other, and the combination can improve the result of the classification process significantly. In the future, a hybrid technique based on clustering algorithms and multilevel thresholdinglike FODPSO can be combined to work on input data set for better results. 


\section{References}

1. S. A. Begum, O. M. Devi, "Fuzzy Algorithms for Pattern Recognition in Medical Diagnosis," Assam University Journal of Science \& Technology: Vol. 7, No. 2, PP. 1-12, 2011.

2. C. H. Bindu, Qiscet, Ongole, "An Improved Medical Image Segmentation Algorithm Using OTSU Method," International Journal of Recent Trends in Engineering, Vol. 2, PP. 88-90, November 2009.

3.A. Javed, W. Y. Chai, N. K. Thuramaiyer, M. S. Javed, and A. R. Alenezi, "Automated Segmentation of Brain MR Images by CombiningContourletTransform and K-Means ClusteringTechniques," In Journal of Theoretical and Applied Information Technology, Vol. 54, PP. 82-91, 10th August 2013.

4. Z. Gang, Z. Dan, H. Ying, H. Xiaobo, Z. Yong, L. Weishi, Z. Yang, Q. Dongxiang, Li Jun, and H. Jiaming," An Unsupervised Method for Brain MRI Segmentation", International Journal of Emerging Technology and Advanced Engineering, Vol. 3,PP.704-709, October 2013.

5. X. Jin,"Multi-Spectral MRI Brain Image Segmentation Based on Kernel Clustering Analysis", International Conference on System Engineering and Modeling, Vol. 34,PP.141- 146, 2012.

6. A. F. Ajala,A. O. Oke,M. O. Alade,A. E. Adewusi,"Fuzzy k-c-means Clustering Algorithm for Medical Image Segmentation," Journal of Information Engineering and Applications, Vol. 2, , PP. 21-32, 2012.

7. E. A. Zanaty, "Improved region growing method for magnetic resonance images (MRIs) segmentation," American Journal of Remote Sensing, Vol. 1, PP. 53-60, 2013.

8. E. Abdel Maksoud, M. Elmogy, R. M. Al-Awadi, "Efficient Hybrid Clustering Techniques for Brain Magnetic Resonance Image Segmentation," In the proceedings of the 2nd International Conference on Advanced Machine Learning Technologies and Applications (AMLTA14), Cairo, Egypt, Nov. 2014.

9. T. S. Madhulatha, "An Overview on clustering methods", International Organization of Scientific Research Journal of Engineering, vol. 2, no. 4, pp. 719-725, Apr. 2012.

10. . J. Acharya1, S. Gadhiya, K. Raviya, "Segmentation Techniques for Image Analysis: A review." International Journal of Computer Science and Management Research, Vol. 2, No. 1, PP. 12181221, January 2013.

11. P. Ghamisi, M. S. Couceiro, J.A. Benediktsson, N. M.F. Ferreira,"Multilevel Image Segmentation Based on Fractional-Order Darwinian Particle Swarm Optimization" IEEE TRANSACTIONS ON GEOSCIENCE AND REMOTE SENSING, PP. 1-13,2013.

12. P. Ghamisi, M. S. Couceiro, J.A. Benediktsson, and N. M.F. Ferreira, "An efficient method for segmentation of images based on fractional calculus and natural selection", Expert Systems with Applications, Vol.39, PP.12407-12417, 2012.

13. P. Ghamisi, M. S. Couceiro, J.A. Benediktsson, N. M.F. Ferreira,'Integration of Segmentation Techniques for Classification of Hyperspectral Images", IEEE GEOSCIENCE AND REMOTE SENSING LETTERS, VOL. 11, PP. 342-346, JANUARY 2014.

14. V. B. Padole, D. S. Chaudhari, "Detection of Brain Tumor in MRI Images Using Mean Shift Algorithm and Normalized Cut Method", International Journal of Engineering and Advanced Technology (IJEAT), Vol. 1, PP. 53-56,June 2012.

15. Q. Mahmood, A. Chodorowski.,A.Mehnert,"A Novel Bayesian Approach to Adaptive Mean Shift Segmentation of Brain Images" IEEE Symposium on Computer-Based Medical Systems, PP. 1 6,2012 . 
16. F. Hamdaoui, A. Sakly, A. Mtibaa,"An efficient multithresholding method for image segmentation based on PSO", International Conference on Control, Engineering \& Information Technology (CEIT'14), PP.203-213, 2014.

17. N. Mangala and B. Suma, "FLGMM algorithm for brain MR image segmentation", International Journal of Latest Research in Science and Technology, Vol. 2,No. 2, PP. 147-152, March -April 2013.

18. S. Bandhyopadhyay, T. Paul, "Automatic Segmentation of Brain Tumour from Multiple Images of Brain MRI" International Journal of Application or Innovation in Engineering \& Management, Vol. 2, no. 1, PP. 240-248, January 2013.

19. E. Abdel-Maksoud, M. Elmogy, R. Al-Awadi ,"Brain tumor segmentation based on a hybrid Clustering technique", Egyptian Informatics Journal , PP 1:11, 10 February 2015.

20. E. Abdel-Maksoud, M. Elmogy, R. Al-Awadi, "MRI Brain Tumor Segmentation System Based on Hybrid Clustering Techniques", Communications in Computer and Information Science, Vol. 488, PP. 401-412, 2014.

21. I. Rodrigues, J. Sanches, J. Dias, “Denoising of Medical Images corrupted by Poisson Noise”. In: 15th IEEE International Conference on Image Processing ICIP, PP. 1756-1759. IEEE Press, San Diego (2008).

22. S. S. Kumar, A. E. Jeyakumar, K.N. Vijeyakumar, N.K. Joel, An adaptive threshold intensity range filter for removal of random value impulse noise in digital images. J. Theoretical Appl. Info. Technol. Vol. 59, PP. 103-112, 2014.

23. Medical image processing analysis and visualization,

ttp://mipav.cit.nih.gov/pubwiki/index.php/Extract_Brain:_Extract_Brain_Surface_

(BET) (Last accessed on 27/03/2015)

24. MICCA nice 2012: http://www2.imm.dtu.dk/projects/BRATS2012/data.html (Lasted accessed on 9/08/2014).

25. Simulated Brain Database, McConnell Brain Imaging Centre, Montreal Neurological Institute, McGill University: http://www.bic.mni.mcgill.ca/brainweb (Lasted accessed on 27/06/2014)

26. K. H. Zou, S. K. Warfield, A. Bharatha, C. M. Tempany, M. R. Kaus, S. J. Haker, W. M. Wells, and R. Kikinis, "Statistical validation of image segmentation quality based on a spatial overlap index," Acad Radiol, Vol. 11, PP. 178-89, 2004.

27. R. Agrawal, M. Sharma, "Review of Segmentation Methods for Brain Tissue with Magnetic Resonance Images",Computer Network and Information Security, Vol. 4, pp. 55-62, 2014. 